\title{
Collagenous Spherulosis Associated with Lobular Carcinoma In Situ of the Breast: Two Case Reports
}

\author{
Ga-Eon Kim • Nah Ihm Kim · Ji Shin Lee · Min Ho Park ${ }^{1}$ \\ Departments of Pathology and 'S Surgery, Chonnam National University Medical School, Gwangju, Korea
}

Collagenous spherulosis (CS) of the breast is an uncommon benign lesion, which is characterized by nodules of eosinophilic or basophilic basement membrane material enclosed in spherical spaces of cribriform architecture with myoepithelial and epithelial proliferation. ${ }^{1-4}$ While CS is usually encountered as an incidental finding in association with other benign hyperplastic lesions, the presence of calcification or abnormal density on imaging studies attracts clinical attention. ${ }^{2-4} \mathrm{CS}$ is occasionally associated with lobular carcinoma in situ (LCIS). ${ }^{3-6}$ Despite its benign nature, the diagnosis of CS is challenging because it shows a cribriform architecture mimicking adenoid cystic carcinoma on low power. ${ }^{3,4,7,8}$ In addition, the diagnosis is especially difficult when associated with LCIS because it is often misdiagnosed as a cribriform pattern ductal carcinoma in situ (DCIS). ${ }^{3-8}$ Recently, it is predicted that the incidence of CS will increase due to breast cancer screening programs, and the pathologists need to be aware of CS in order not to misdiagnose CS associated with LCIS as DCIS or other malignancy, especially on core needle biopsy specimens. Here, we report two cases of CS associated with LCIS of the breast. To the best of our knowledge, this is the first reported case of CS associated with LCIS of the breast in Korea.

\section{CASE REPORT}

The cases reported herein were consult cases, and this study was approved by the Institutional Review Board of Chonnam

\section{Corresponding Author}

Ji Shin Lee, MD

Department of Pathology, Chonnam National University Hwasun Hospital,

322 Seoyang-ro, Hwasun 58128, Korea

Tel: +82-61-379-7072, Fax: +82-61-379-7079, E-mail: jshinlee@hanmail.net

Received: January 23, 2018 Revised: March 19, 2018

Accepted: March 27, 2018
National University Hwasun Hospital with waiver of informed consents (CNUHH-EXP-2018-012).

Case 1 was an asymptomatic 51-year-old female with multiple small nodules on ultrasound examination of the right breast. Case 2 was a 47-year-old female with an incidentally detected lesion in the left breast presenting as an ill-defined nodule measuring $8 \mathrm{~mm}$ on ultrasound examination. Both patients underwent ultrasound-guided vacuum-assisted biopsy. The histology of the two cases was similar. Microscopically, the lesions showed enlarged lobules filled with neoplastic cells (Figs. 1A, 2A). The neoplastic cells of case 1 contained scant cytoplasm, and small, rounded, and bland nuclei without nucleoli (Fig. 1B). In case 2, the neoplastic cells showed more abundant cytoplasm than those seen in case 1, and larger, more pleomorphic nuclei with nucleoli (Fig. 2B). The neoplastic cells in both cases showed incohesive growth and negative E-cadherin immunoreactivity. Cases 1 and 2 represented classical and pleomorphic types of LCIS, respectively. In both cases, multiple spherules showing cribriform architecture lined by flattened epithelial cells were seen adjacent to the LCIS area (Figs. 1C, 2C). The space of the spherules contained faintly basophilic fibrillary substances. Overall, the spherules displayed microscopic characteristics typical of CS. A few spherules of CS had LCIS (Figs. 1D, 2D). E-cadherin-negative LCIS cells colonized several spherules of CS and replaced the luminal epithelial cells, which were positive for E-cadherin (Figs. 1E, 2E). The spherules were outlined by myoepithelial cells stained with p63 and calponin (Figs. 1F, 2F, G). The basophilic fibrillary materials within CS were positive for laminin (Fig. 1G). The cells of the spherules tested negative for c-Kit (Figs. 1H, 2H). 


\section{DISCUSSION}

CS is a rare breast lesion with well-characterized, structural, and histological alterations of unknown histogenesis. ${ }^{1-4}$ Histologically, CS constitutes less than $1 \%$ of all breast biopsies. ${ }^{2,4}$ Typically, CS is detected as an incidental microscopic finding in
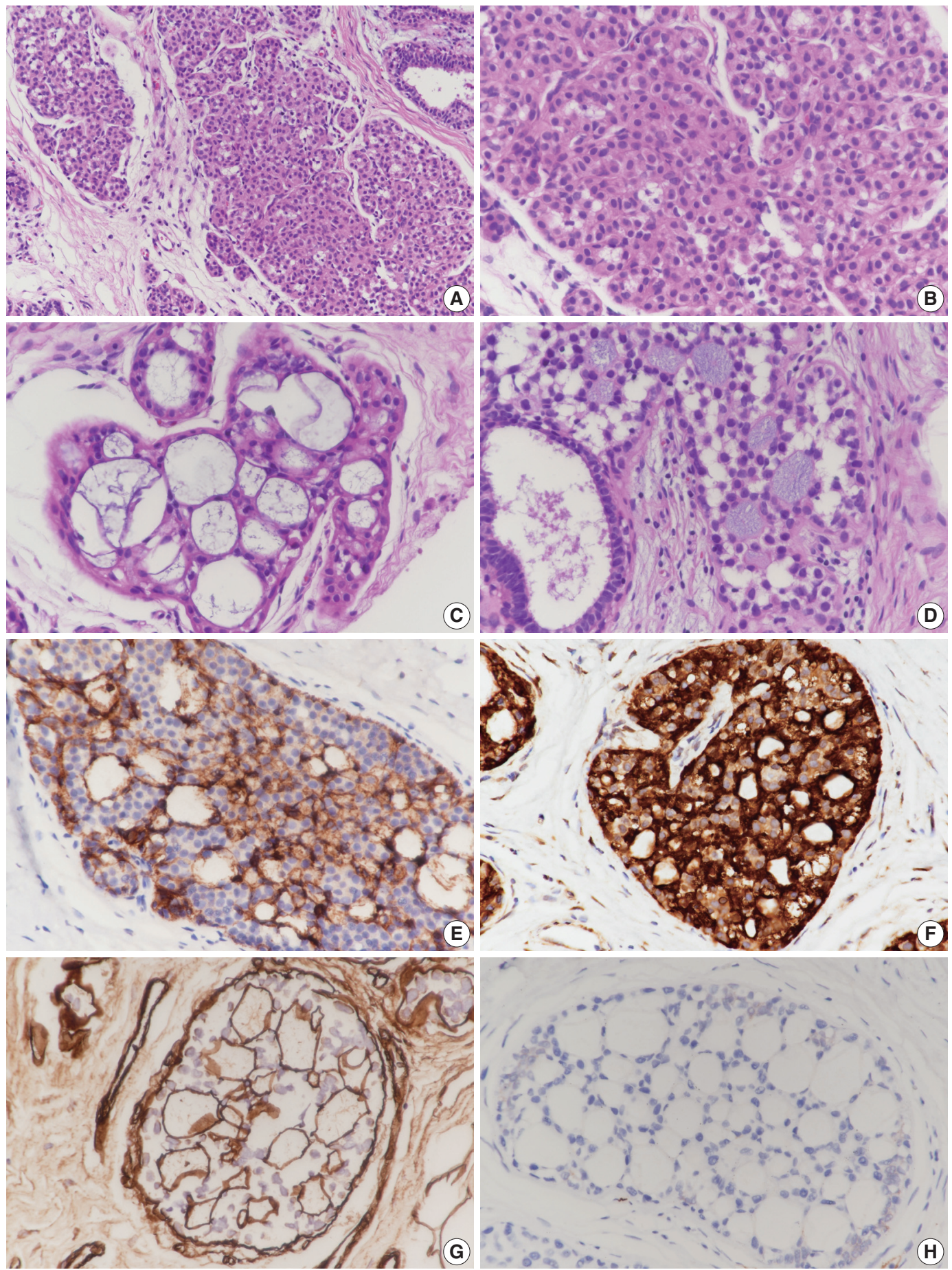

Fig. 1. Microscopic and immunohistochemical findings of case 1 collagenous spherulosis (CS) associated with lobular carcinoma in situ (LCIS), classical type. (A) In LCIS, enlarged lobules are seen. (B) The neoplastic cells of LCIS show loss of cohesion. (C) Cribriform proliferation with spherules containing cellular fibrillar components is seen. (D) LCIS cells colonize CS. (E) LCIS cells stain negative for E-cadherin, and the residual cells of CS stain positive. (F) Myoepithelial cells within CS with LCIS show calponin immunoreactivity. (G) Basement membrane-like components within spherules are highlighted by laminin immunostain. $(\mathrm{H}) \mathrm{CS}$ with LCIS is negative for c-Kit. 

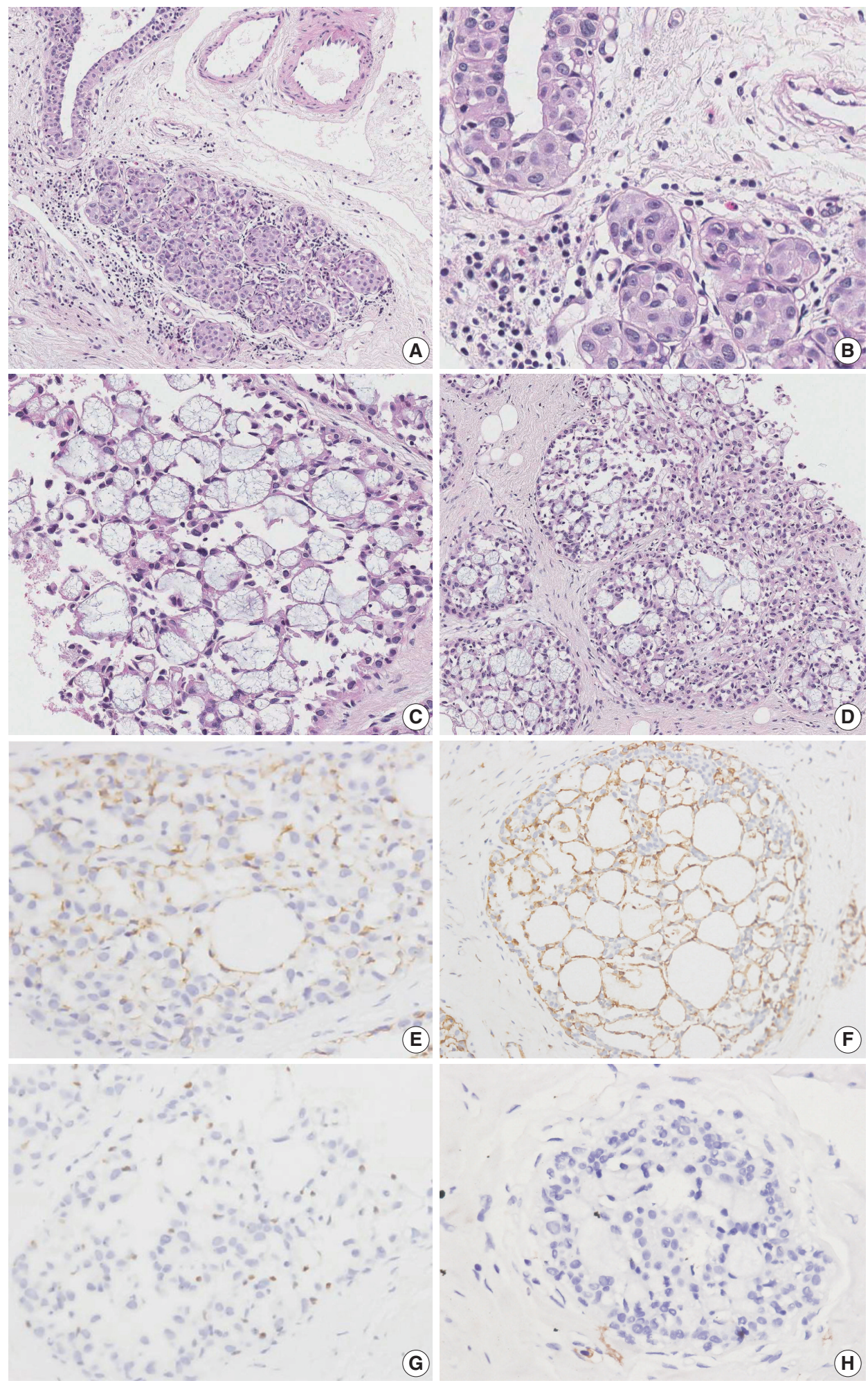

Fig. 2. Microscopic and immunohistochemical findings of case 2 collagenous spherulosis (CS) associated with lobular carcinoma in situ (LCIS), pleomorphic type. (A) The LCIS area shows enlarged lobular glands and intraepithelial growth pattern. (B) In contrast to case 1, the neoplastic cells contain more abundant cytoplasm and pleomorphic nuclei with occasional nucleoli. (C) A cribriform pattern of CS is characterized by cystic spaces containing basophilic fibrillar components. (D) CS with LCIS retains cribriform spaces, which contain cellular fibrillar components. (E) LCIS cells within the spherule show loss of E-cadherin expression. (F, G) The cells surrounding the spherules are positive for calpoinin and p63. (H) CS with LCIS is negative for c-Kit. 
surgical specimens of other lesions. CS can also be detected as calcifications or an abnormal density on imaging studies. ${ }^{3,4}$ Benign proliferative lesions associated with CS include papillomas, ductal hyperplasia, radial scars, and complex sclerosing lesions. ${ }^{3,4} \mathrm{CS}$ is occasionally associated with LCIS. ${ }^{3-6}$ The prognosis and treatment depend on the nature of the underlying lesion.

Eisenberg and Hoda ${ }^{6}$ summarized the clinical and morphological features of 38 cases of CS diagnosed with LCIS at a single institution over a 12 -year period. All the cases were submitted for consultation either for diagnostic assistance or with the mistaken interpretation of DCIS. The patients included women ranging in age from 41 to 75 years (mean age, 52 years). CS with LCIS was demonstrated in 22 core-needle biopsy specimens (58\%), 15 excisional specimens (40\%), and 1 mastectomy specimen (2\%). Both breasts were equally involved and no case was bilateral. Thirty-four cases (89\%) presented with mammographically detected abnormal density, with associated microcalcifications in 24 (63\%). Histologically, CS with LCIS showed expanded ducts and glandular acini with relatively uniform cribriform spaces, which contained fibrillary or stellate basement membrane material. LCIS and CS were seen immediately adjacent to CS with LCIS. The constituent LCIS in CS with LCIS represented the classical type in 35 cases (92\%), pleomorphic type in one (3\%) and a mixture of classical and pleomorphic type in two cases $(5 \%)^{6}$

To the best of our knowledge, the cases reported herein represent the first cases of CS with LCIS in Korea. Both of our patients were female, aged 47 and 51 years, and the lesions were detected incidentally by ultrasound screening. The lesions showed LCIS, one a classical type and the other a pleomorphic type.

With experience, the recognition of CS with LCIS may not be challenging, but it can still pose a diagnostic difficulty. The most common lesion for which CS with LCIS may be mistaken is adenoid cystic carcinoma. ${ }^{3,4,7,8}$ Both lesions contain spherules with basement membrane materials. The spherules in CS with LCIS and adenoid cystic carcinoma are similar in composition; histochemical and immunohistochemical studies show components of basement membrane, including type IV collagen and laminin, in both lesions. Functional myoepithelial cells are known to generate basement membrane components, which form spherical masses. However, the two lesions can be distinguished by the growth pattern; CS with LCIS is not an infiltrative lesion while adenoid cystic carcinoma shows stromal invasion. In challenging cases, immunohistochemical stains can facilitate differential diagnosis. ${ }^{4,7-10}$ Immunostaining for myoepithleial markers such as smooth muscle myosin heavy chain, p63, and calponin demonstrate the presence of a single layer of myoepithelial cells surround- ing the spherules in CS. Variable expression of myoepithelial cell markers is reported due to the basal/myoepithelial phenotype of the tumor cells in adenoid cystic carcinoma,. Additionally, adenoid cystic carcinoma shows positive staining for c-Kit, unlike CS with LCIS. $^{9,10}$

CS with LCIS imparts a regular, chiseled cribriform architecture with monotonous neoplastic cells, which can be mistaken for low grade DCIS of cribriform pattern. ${ }^{3-8}$ Recognition of the incohesive growth and punctate cytoplasmic vacuoles of the LCIS cells also facilitates an accurate diagnosis. Immunostaining for E-cadherin in combination with myoepithelial cell makers can further differentiate CS with LCIS from DCIS. In CS with LCIS, E-cadherin is negative in the neoplastic LCIS cells and positive in the intermingled residual epithelial and myoepithelial cells of CS. Myoepithelial cell immunostaining demonstrates the presence of myoepithelial cells within the spherules. In DCIS, E-cadherin is positive in the neoplastic epithelial cells, and myoepithelial cell markers highlight the peripheral myoepithelial cell layer.

Lobular neoplasia includes atypical lobular hyperplasia and LCIS, and the distinction between the two is based on the degree of acinar involvement in a lobular unit. In core-needle biopsy specimens, CS involved by lobular neoplasia is a more appropriate diagnostic term.

We diagnosed CS with LCIS based on results of immunohistochemical studies. E-cadherin-negative LCIS cells colonized the spherules in CS, surrounded by a layer of myoepithelial cells stained with $\mathrm{p} 63$ and calponin. The tumor cells tested negative for c-Kit.

In conclusion, breast cancer screening programs may detect increased numbers of CS cases with LCIS or CS alone. The pathologist should be aware of this lesion to avoid erroneous diagnosis of malignancy, especially in core-needle biopsy specimens.

\section{ORCID}

Ji Shin Lee: https://orcid.org/0000-0003-4634-2228

\section{Conflicts of Interest}

No potential conflict of interest relevant to this article was reported.

\section{REFERENCES}

1. Clement PB, Young RH, Azzopardi JG. Collagenous spherulosis of the breast. Am J Surg Pathol 1987; 11: 411-7.

2. Mooney EE, Kayani N, Tavassoli FA. Spherulosis of the breast: a 
spectrum of municous and collagenous lesions. Arch Pathol Lab Med 1999; 123: 626-30.

3. Resetkova E, Albarracin C, Sneige N. Collagenous spherulosis of breast: morphologic study of 59 cases and review of the literature. Am J Surg Pathol 2006; 30: 20-7.

4. Hoda SA, Brogi E, Koerner FC, Rosen PP, eds. Rosen's breast pathology. 4th ed. Philadelphia: Lippincott Williams \& Wilkins, 2014; 143-7.

5. Sgroi D, Koerner FC. Involvement of collagenous spherulosis by lobular carcinoma in situ: potential confusion with cribriform ductal carcinoma in situ. Am J Surg Pathol 1995; 19: 1366-70.

6. Eisenberg RE, Hoda SA. Lobular carcinoma in situ with collagenous spherulosis: clinicopathologic characteristics of 38 cases. Breast
J 2014; 20: 440-1.

7. Toll A, Joneja U, Palazzo J. Pathologic spectrum of secretory and mucinous breast lesions. Arch Pathol Lab Med 2016; 140: 644-50.

8. Torous VF, Schnitt SJ, Collins LC. Benign breast lesions that mimic malignancy. Pathology 2017; 49: 181-96.

9. Cabibi D, Giannone AG, Belmonte B, Aragona F, Aragona F. CD10 and HHF35 actin in the differential diagnosis between Collagenous spherulosis and adenoid-cystic carcinoma of the breast. Pathol Res Pract 2012; 208: 405-9.

10. Rabban JT, Swain RS, Zaloudek CJ, Chase DR, Chen YY. Immunophenotypic overlap between adenoid cystic carcinoma and collagenous spherulosis of the breast: potential diagnostic pitfalls using myoepithelial markers. Mod Pathol 2006; 19: 1351-7. 